\title{
Results on the two nucleon-induced Non Mesonic Weak Decay of $\Lambda$ hypernuclei
}

\author{
Stefania Bufalino* \\ INFN-Sezione di Torino, via P. Giuria 1, Torino, Italy \\ E-mail: bufalino@to.infn.it
}

\section{Agnello}

Dip. di Fisica, Politecnico di Torino, Corso Duca degli Abruzzi 24, Torino, Italy INFN-Sezione di Torino, via P. Giuria 1, 10125 Torino

E-mail: michelangelo.agnello@polito.it

\section{Benussi, M. Bertani, F. L. Fabbri, P. Gianotti, V. Lucherini}

Laboratori Nazionali di Frascati dell'INFN, via E. Fermi 40, Frascati, Italy

E-mail: benussi@lnf.infn.it; monica.bertani@lnf.infn.it;

Franco.Fabbri@lnf.infn.it; gianotti@lnf.infn.it;

vincenzo.lucherini@lnf.infn.it

\section{H.C. Bhang}

Dep. of Physics, Seoul National Univ., 151-742 Seoul, South Korea

E-mail: bhang@phya.snu.ac.kr

\section{G. Bonomi, F. Moia, A. Zenoni}

Dip. di Ingegneria Meccanica e Industriale, Università di Brescia, via Valotti 9, Brescia, Italy INFN Sez. di Pavia, via Bassi 6, Pavia, Italy

E-mail: bonomi@cern.ch; Fabio.Moia@ing.unibs.it;

Aldo.Zenoni@bs.infn.it

\section{E. Botta, T. Bressani, F. De Mori, S. Marcello}

Dip. di Fisica Sperimentale, Università di Torino, via P. Giuria 1, Torino, Italy

INFN-Sezione di Torino, via P. Giuria 1, Torino, Italy

E-mail: botta@to.infn.it; bressani@to.infn.it; demori@to.infn.it; marcellodto.infn.it

\section{Bregant, P. Camerini, R. Rui}

Dip. di Fisica Università di Trieste, via Valerio 2, Trieste, Italy

INFN Sez. di Trieste, via Valerio 2, Trieste, Italy

E-mail: Bregantets.infn.it; cameriniets.infn.it;

rinaldo.rui@ts.infn.it

\section{Busso}

Dip. di Fisica Generale, Università di Torino, via P. Giuria 1, Torino, Italy INFN-Sezione di Torino, via P. Giuria 1, Torino, Italy

E-mail: busso@to.infn.it 


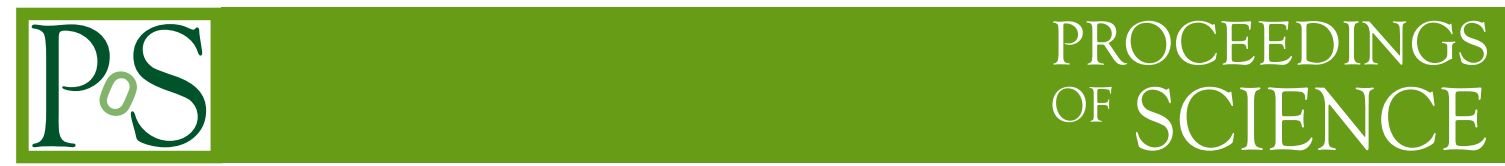

\section{Calvo, A. Feliciello, A. Filippi, R. Wheadon}

INFN-Sezione di Torino, via P. Giuria 1, Torino, Italy

E-mail: calvo@to.infn.it; feliciello@to.infn.it;

filippieto.infn.it; wheadon@to.infn.it

\section{B. Dalena}

CEA, Irfu/SACM, Gif-sur-Yvette, France

E-mail: barbara.dalena@cern.ch

\section{G. D’Erasmo, E. M. Fiore, G. Simonetti}

Dip. InterAteneo di Fisica, via Amendola 173, Bari, Italy

INFN Sez. di Bari, via Amendola 173, Bari, Italy

E-mail: derasmo@ba.infn.it; enrica.fiore@ba.infn.it;

giuseppe.simonetti@ba.infn.it

\section{H. Fujioka, T. Nagae}

Department of Physics, Sakyo-ku, Kyoto 606-8502, Japan

E-mail: fujioka@riken.jp; nagaedscphys.kyoto-u.ac.jp

\section{N. Grion, S. Piano}

INFN Sez. di Trieste, via Valerio 2, Trieste, Italy

E-mail: grionets.infn.it; pianodts.infn.it

\section{N. Mirfakhrai}

Dep. of Physics, Shahid Behesty Univ., 19834 Teheran, Iran

E-mail: mirfakhrai@yahoo.com

\section{P. Montagna}

Dip. di Fisica Teorica e Nucleare, Universita' di Pavia, via Bassi 6, Pavia, Italy

INFN Sez. di Pavia, via Bassi 6, Pavia, Italy

E-mail: Paolo.Montagna@pv.infn.it

\section{O. Morra}

INAF-IFSI Sez. di Torino, C.so Fiume 4, Torino, Italy

INFN-Sezione di Torino, via P. Giuria 1, Torino, Italy

E-mail: Morra@to.infn.it

\section{H. Outa}

RIKEN, Wako, Saitama 351-0198, Japan

E-mail: outa@riken.jp

\section{A. Pantaleo, V. Paticchio}

INFN Sez. di Bari, via Amendola 173, Bari, Italy

New measurements of the proton spectra from Non Mesonic Weak Decay (NMWD), with the FINUDA experiment, triggered a search on the two nucleon-induced $\Lambda n p \rightarrow n n p$ decay. This leads to the determination of $\Gamma_{2} / \Gamma_{N M W D}=0.21 \pm 0.07_{\text {stat }}{ }_{-0.02 \text { sys }}^{+0.03 \text { sys }}$.

8th International Conference on Nuclear Physics at Storage Rings-Stori11,

October 9-14, 2011

Laboratori Nazionali di Frascati, Italy 


\section{Introduction}

The information coming from the study of the $\Lambda$-hypernuclei weak decay channels completes the knowledge on strange nuclear systems by spectroscopy, gained both by missing mass analyses and $\gamma$-ray measurements. $\Lambda$-hypernuclei decay through both mesonic (MWD) and non-mesonic weak decay (NMWD) processes. In MWD the $\Lambda$ hyperon decays to a nucleon and a pion in the nuclear medium, similarly to the weak decay mode in free space:

$$
\begin{aligned}
\Lambda_{\text {free }} \rightarrow & p+\pi^{-}+37.8 \mathrm{MeV} \\
& (64.2 \%) \\
n+\pi^{0}+41.1 \mathrm{MeV} & (35.8 \%)
\end{aligned}
$$

in which the emitted nucleon (pion) carries a momentum $q \approx 100 \mathrm{MeV} / \mathrm{c}$. The theory of hypernuclear MWD was initiated by Dalitz [1,2], and motivated by the observation of MWD reactions in the pioneering hypernuclear physics experiments with photographic emulsions. Following the development of counter techniques for use in $\left(K^{-}, \pi^{-}\right)$and $\left(\pi^{+}, K^{+}\right)$reactions on nuclei in the 1970s and 1980s, a considerable body of experimental data on $\Gamma_{\pi^{-}}$and/or $\Gamma_{\pi^{0}}$ is now available on light $\Lambda$-hypernuclei up to ${ }_{\Lambda}^{12} \mathrm{C}$ ([3] and references therein).

In NMWD the $\Lambda$-Hypernucleus decays through processes which involve a weak interaction of the constituent $\Lambda$ with one or more core nucleons. The importance of such a process was pointed out just at the beginning of hypernuclear physics: Cheston and Primakoff gave a quantitative discussion of the possibility that a $\Lambda$-hyperon bound to nucleons might undergo NMWD in 1953 [4]. If the pion emitted in the hadronic vertex $\Lambda \rightarrow \pi N$ is virtual, then it can be absorbed by the nuclear medium, resulting in a non-mesonic decay of the following types:

$$
\begin{aligned}
& { }_{\Lambda}^{A} \mathrm{Z} \rightarrow{ }^{A-2}(\mathrm{Z}-1)+p+n \quad\left(\Gamma_{p}\right) \\
& { }_{\Lambda}^{A} \mathrm{Z} \rightarrow{ }^{A-2} \mathrm{Z}+n+n \quad\left(\Gamma_{n}\right) \\
& { }_{\Lambda}^{A} \mathrm{Z} \rightarrow{ }^{A-3} \mathrm{Z}^{\prime}+N+N+N \quad\left(\Gamma_{2}\right)
\end{aligned}
$$

The channel (5) is known as two-nucleon induced (2N) decay and is due to the interaction of the $\Lambda$ with a pair of strongly correlated nucleons; $Z^{\prime}$ stands for $Z, Z-1$ or $Z-2$ depending on the particular nucleons combination. The total NMWD rate of a $\Lambda$-hypernucleus is given by their sum: $\Gamma_{\mathrm{NMWD}}=\Gamma_{p}+\Gamma_{n}+\Gamma_{2}$. Several important experimental progresses in NMWD study have been made in the latest years but no experimental evidence has been obtained so far for the $2 \mathrm{~N}$ induced decay. In the present work we report on measurements by the FINUDA experiment and we will describe two independent analyses of the NMWD of ${ }_{\Lambda}^{7} \mathrm{Li},{ }_{\Lambda}^{9} \mathrm{Be},{ }_{\Lambda}^{11} \mathrm{~B},{ }_{\Lambda}^{12} \mathrm{C},{ }_{\Lambda}^{13} \mathrm{C},{ }_{\Lambda}^{15} \mathrm{~N}$ and ${ }_{\Lambda}^{16} \mathrm{O}$ hypernuclei which provided values for the $2 \mathrm{~N}$ decay. The complete analysis and discussion of the results was already published in Ref. [5, 6].

\section{Analysis method and results}

The results reported in the present paper have been obtained by analyzing the data collected by the FINUDA experiment, from 2003 to 2007 and correspond to an integrated luminosity of

\footnotetext{
*Speaker.

$\dagger$ deceased
} 
$1.2 \mathrm{fb}^{-1}$. FINUDA is a fixed target experiment installed at one of the two interaction points of the DA $\Phi \mathrm{NE} e^{+} e^{-} \phi$-factory of Laboratori Nazionali di Frascati (INFN-Italy). $\Lambda$-hypernuclei are produced by means of the $\left(K^{-}, \pi^{-}\right)$reaction with $K^{-}$'s at rest by stopping in very thin targets the low energy $(\sim 16 \mathrm{MeV}) K^{-}$'s coming from the $\Phi \rightarrow K^{-} K^{+}$decay channel. FINUDA was a magnetic spectrometer immersed in a uniform solenoidal magnetic field of $1 \mathrm{~T}$. The tracker included a combination of silicon microstrips (OSIM), low mass drift chambers (LMDC) and a stereo system of straw tubes (ST). It was optimized for the detection of charged particles featuring a momentum resolution $<1 \%$ FWHM for $\pi^{-}$of $250-290 \mathrm{MeV} / \mathrm{c}$, corresponding to the formation of hypernuclear bound states, and an energy resolution for $p$ ranging from $1.3 \%$ to $4 \%$ FWHM for kinetic energies from $90 \mathrm{MeV}$ down to $50 \mathrm{MeV}$ [5]. Its angular acceptance was $2 \pi$ sr. OSIM and LMDC were used also for P.Id. of the charged particles. The inner FINUDA detector was an hodoscope of thin scintillator slabs (TOFINO) arranged around the beam pipe at the $\left(e^{+}, e^{-}\right)$interaction point. An array of silicon microstrips (ISIM) was located outside TOFINO, very near to the targets, to determine the position of the $K^{ \pm}$interaction point. An array of eight target tiles was located between ISIM and the tracker. The outer FINUDA detector, called TOFONE, was a plastic $(\mathrm{CH})_{n}$ scintillator barrel $(10 \mathrm{~cm}$ thick) surrounding the tracker. It was also used to detect neutrons (and photons) with an efficiency of about $10 \%$ for neutrons in the energy range $15-150 \mathrm{MeV}$. The particles Time-Of-Flight (TOF) was measured by the (TOFINO-TOFONE) system. The analysis was performed on events collected out of ${ }^{6} \mathrm{Li},{ }^{7} \mathrm{Li},{ }^{9} \mathrm{Be},{ }^{12} \mathrm{C},{ }^{13} \mathrm{C}$ and $\left(\mathrm{D}_{2} \mathrm{O}\right)$ targets. The experimental method is briefly described here, while full details are reported in $[5,6]$. To investigate the NMWD process all the events characterized by the presence of two particles emitted in coincidence were selected: a $\pi^{-}$with a momentum as high as $260-290 \mathrm{MeV} / \mathrm{c}$, gives the signature of the formation of the ground-state of the hypernuclear system or of a low lying excited state decaying to it by electromagnetic emission and a proton coming from the same $K^{-}$interaction vertex, which gives the signature of the NMWD. Protons were identified with an efficiency of $90 \%$ and the proton energy resolution was $\Delta \mathrm{E} \sim 2 \% \mathrm{FWHM}$ at $80 \mathrm{MeV}$. A further requirement was made asking for the detection of a neutron. The outer FINUDA detector, called TOFONE [6], a barrel of 72 scintillator slabs was used for trigger and P.Id. (by Time Of Flight) of the charged particles and to detect even neutral ones with an efficiency of the order of $10 \%$ for neutrons in the range $15-150 \mathrm{MeV}$. The energy resolution on the neutron is $\sim 13 \%$ at $10 \mathrm{MeV}$ and $\sim 10 \%$ at $100 \mathrm{MeV}$. With regard to the NMWD study, in Fig. 2 the kinetic energy spectra of protons coming from the NMWD of ${ }_{\Lambda}^{5} \mathrm{He}$, ${ }_{\Lambda}^{7} \mathrm{Li},{ }_{\Lambda}^{9} \mathrm{Be},{ }_{\Lambda}^{11} \mathrm{~B},{ }_{\Lambda}^{12} \mathrm{C},{ }_{\Lambda}^{13} \mathrm{C},{ }_{\Lambda}^{15} \mathrm{~N}$ and ${ }_{\Lambda}^{16} \mathrm{O}$ are shown. All the spectra are background subtracted and acceptance corrected; the errors in the spectra are statistical only and include both the contributions from background subtraction and acceptance correction. We estimated a systematic error of less than 5\%. Even though affected by considerable errors, in particular in the low energy region, the proton kinetic energy spectra show a clear trend as a function of the hypernuclear mass number $A$ (from 5 to 16): a peak around $80 \mathrm{MeV}$ (which corresponds to about half of the $Q$-value for the free $\Lambda p \rightarrow n p$ reaction) is broadened by the Fermi motion of nucleons and more and more blurred as $A$ increases. The peak is smeared, on its low energy side, by a rise that can be ascribed to FSI and two-nucleon induced weak decays.

To estimate the contribution of this last channel each proton spectrum from $80 \mathrm{MeV}$ onwards was fitted by a Gaussian function. It was assumed that the proton spectrum beyond the peak mean value is due to protons coming from the $\Lambda p \rightarrow n p$ reaction and that the $2 \mathrm{~N}$ channel can be neglected in 
this region $\left(\mathrm{A}_{\text {high }}\right)$. On the contrary, the spectrum below the peak mean value is fed by protons from both $\Lambda p \rightarrow n p$ and $2 \mathrm{~N}$ decays and is also affected by FSI $\left(\mathrm{A}_{\text {low }}\right)$.

The determination of the $2 \mathrm{~N}$ decay was based on two assumptions: the first one is that the twonucleon induced NMWD is dominated by the $\Lambda n p \rightarrow n n p$ channel $\left(\Gamma_{2}=\Gamma(\Lambda n p \rightarrow n n p)+\right.$ $\left.\Gamma(\Lambda p p \rightarrow n p p)+\Gamma(\Lambda n n \rightarrow n n n) \equiv \Gamma_{n p}+\Gamma_{p p}+\Gamma_{n n} \simeq \Gamma_{n p}\right)$ since the recent microscopical calculation of Ref. [11] quote $\Gamma_{n p}: \Gamma_{p p}: \Gamma_{n n}=0.83: 0.12: 0.04$ and the second one stands on a constant $\Gamma_{2} / \Gamma_{p}$ ratio for the considered hypernuclear mass range.
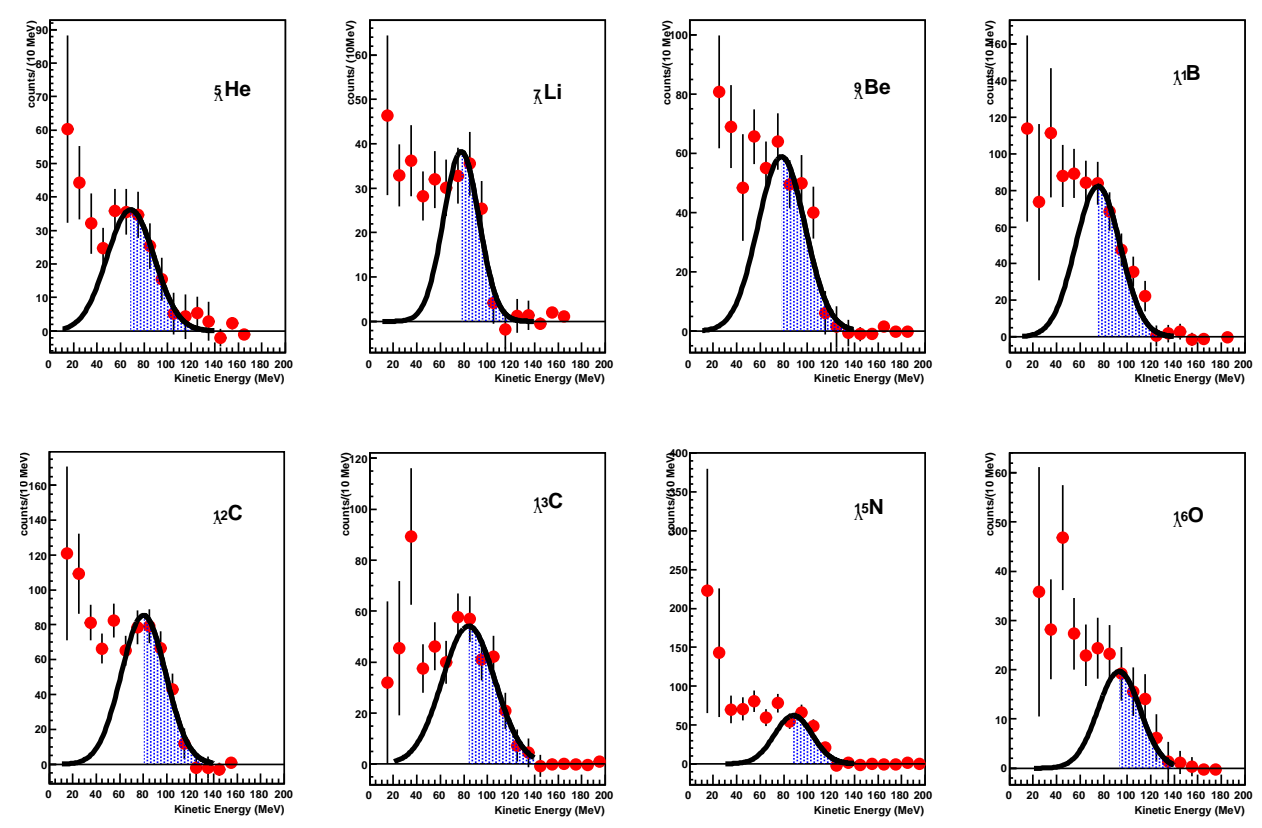

Figure 1: Proton kinetic energy spectra from the NMWD of (from left to right): ${ }_{\Lambda}^{5} \mathrm{He},{ }_{\Lambda}^{7} \mathrm{Li},{ }_{\Lambda}^{9} \mathrm{Be},{ }_{\Lambda}^{11} \mathrm{~B},{ }_{\Lambda}^{12} \mathrm{C}$, ${ }_{\Lambda}^{13} \mathrm{C},{ }_{\Lambda}^{15} N$ and ${ }_{\Lambda}^{16} \mathrm{O}$. The blue filled area is the spectrum area in which the two-nucleon induced NMWD is negligible.

Consider now the ratio:

$$
R \equiv \frac{A_{\text {low }}}{A_{\text {low }}+A_{\text {high }}}=\frac{0.5+\frac{\Gamma_{2}}{\Gamma_{p}}}{1+\frac{\Gamma_{2}}{\Gamma_{p}}}+b A .
$$

In Fig. 2 the experimental values of this ratio are plotted as a function of A.

Applying a model independent analysis, fully described in [5] a value of $\Gamma_{2} / \Gamma_{p}=0.43 \pm 0.25$ was determined. As discussed in [5], to determine $\Gamma_{2} / \Gamma_{\mathrm{NMWD}}$ the $\Gamma_{n} / \Gamma_{p}$ ratio needs to be known. Using recent experimental results [8] for ${ }_{\Lambda}^{5} H$ and for ${ }_{\Lambda}^{12} \mathrm{C}$ together with the above determination of $\Gamma_{2} / \Gamma_{p}$ one obtains $\Gamma_{2} / \Gamma_{\mathrm{NMWD}}=0.24 \pm 0.10$. In a following analysis of the events with triple $\left(\pi^{-}, \mathrm{n}, \mathrm{p}\right)$ coincidences a neutron was required in addition. We also fixed for each hypernucleus a proton energy threshold $E_{p}=20 \mathrm{MeV}$ below the Gaussian mean value $\mu$ found in [5]. We verified that this value was the best compromise on the signal/background ratio and furthermore we chose 


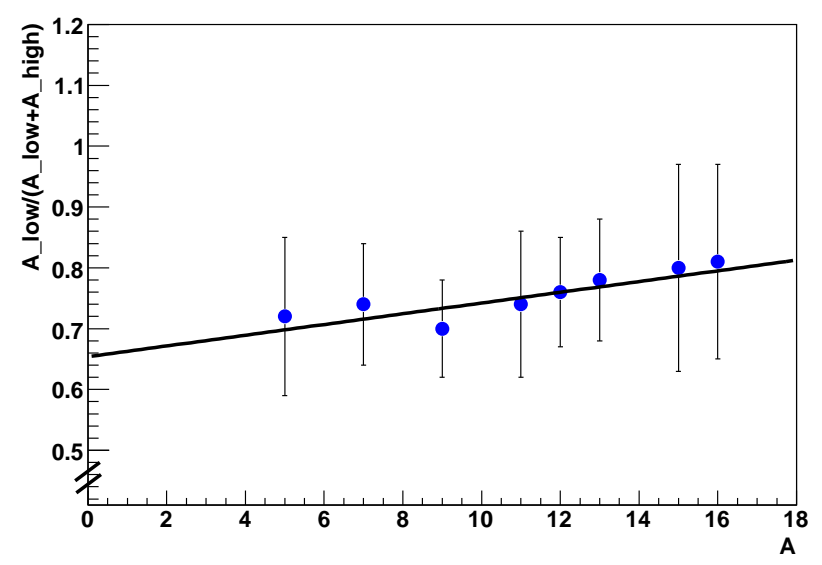

Figure 2: The ratio $A_{\text {low }} /\left(A_{\text {low }}+A_{\text {high }}\right)$ as a function of the hypernuclear mass number.

a threshold for the angular correlation between the neutron and the proton of $\cos \theta(\mathrm{np})=-0.8$.

We analized then triple $\left(\pi^{-}, \mathrm{n}, \mathrm{p}\right)$ coincidences selecting all the events with $E_{p}$ lower than the threshold and $\cos \theta(n p)>-0.8$. These events correspond mainly to the $\Lambda n p \rightarrow n n p$ process with a small contribution of FSI.

For each nucleus the following ratio was then considered:

$$
R_{2}(A) \equiv \frac{N_{n p}\left(E_{p} \leq E_{p}^{\mathrm{thr}}, \cos \theta_{n p} \geq-0.8\right)}{N_{p}\left(E_{p}>\mu\right)}=\frac{0.8 \Gamma_{n p}}{0.5 \Gamma_{p}}+b A,
$$

where the numerator of the first fraction indicates the number of $n p$ events satisfying the condition which selects two-nucleon induced candidates and $N_{p}\left(E_{p}>\mu\right)$ is the number of protons in the part of the spectrum of Ref. [5] above the Gaussian mean value.

Fig. 3 shows the dependence of $R_{2}(A)$ on the Hypernuclear mass number. Also in this case it is possible to apply the same considerations on the $A$-dependence of FSI and $\Gamma_{2}$ used for the study of the single proton spectra.

Using the values of $R_{2}(A)$, obtained for each hypernucleus, we get:

$$
\frac{\Gamma_{n p}}{\Gamma_{p}}=0.39 \pm 0.16_{\text {stat }}{ }_{-0.03 \text { sys }}^{+0.04} .
$$

Using the experimental value of $\Gamma_{n} / \Gamma_{p}$ reported in [8] for ${ }_{\Lambda}^{5} \mathrm{He}$ and ${ }_{\Lambda}^{12} \mathrm{C}$ and our determination of $\Gamma_{n p} / \Gamma_{p}$ we obtain $\Gamma_{2 N} / \Gamma_{\mathrm{NMWD}}=0.21 \pm 0.07_{\text {stat }}{ }_{-0.02_{\text {sys }}}^{+0.00}$. This value supports the latest theoretical predictions [13] $\left(\Gamma_{2} / \Gamma_{\mathrm{NMWD}}=0.26\right)$, the recent experimental results of [10] $(0.29 \pm 0.13)$ and the previous FINUDA result [5], but bears a smaller error. Calculating the weighted mean of the experimental determinations $[6,10]$ of $\Gamma_{2 N} / \Gamma_{\mathrm{NMWD}}$ at our disposal one found that the contribution of the two-nucleon induced decay is $0.23 \pm 0.06$.

\section{Conclusions}

The FINUDA experiment has performed a systematic study of NMWD charged particles spectra of $\Lambda$-hypernuclei in the $A=5 \div 16$ mass range. The kinetic energy spectra of protons from the 


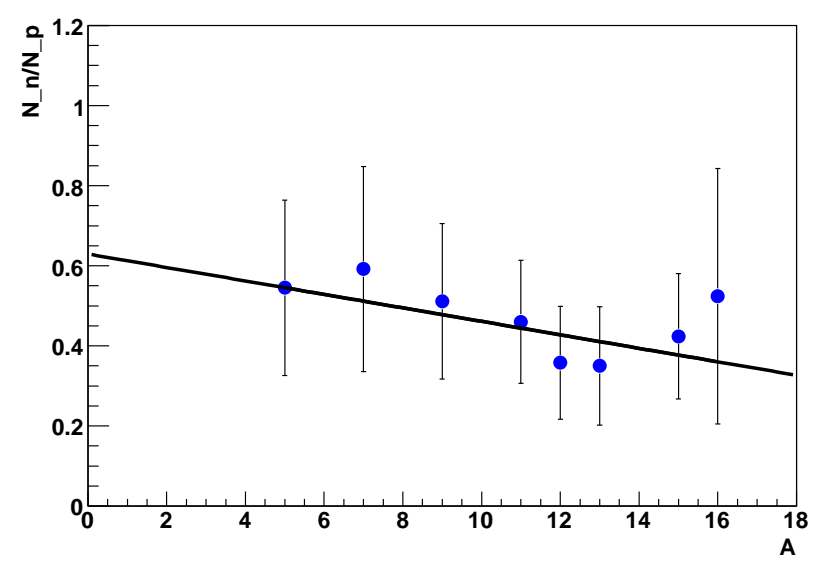

Figure 3: The ratio $N_{\mathrm{n}} / N_{\mathrm{p}}$ as a function of the hypernuclear mass number A.

NMWD were analyzed and it was possible to disentangle the nucleon FSI effects and the $2 \mathrm{~N}$ decay contribution to the NMWD process: an experimental value of $\Gamma_{2} / \Gamma_{N M W D}=0.24 \pm 0.10$ was obtained. This result was confirmed in the analysis of the triple $\left(\pi^{-}, n, p\right)$ coincidences, with a reduction of the error and leading to an estimate of $\Gamma_{2} / \Gamma_{N M W D}=0.21 \pm 0.07_{\text {stat }}+0.0 .03_{\text {sys }}$

\section{References}

[1] R.H. Dalitz, L. Liu, Phys. Rev. 116 (1959) 1312.

[2] D. Kiełczewska, D. Ziemińska, R.H. Dalitz, Nucl. Phys. A 333 (1980) 367.

[3] Y. Sato, et al., Phys. Rev. C 71 (2005) 025203.

[4] Cheston W and Primakoff H (1953) Phys. Rev. 921537.

[5] Agnello M et al., (2010) Phys. Lett. B 685247.

[6] Agnello M et al., (2011) Phys. Lett. B 701556.

[7] T. Motoba, K. Itonaga, Progr. Theor. Phys. Suppl. 117 (1994) 477.

[8] Bhang H et al., (2007) Eur. Phys. J. A 33259.

[9] Parker J D et al., (2007) Phys. Rev. C 76035501.

[10] Kim M et al., (2009) Phys. Rev. Lett. 103182502.

[11] Bauer E and Garbarino G, (2009) Nucl. Phys. A 82829.

[12] Agnello M et al., (2008) Nucl. Phys. A 804151.

[13] E. Bauer and G. Garbarino, Phys. Rev C 81 (2010) 064315. 